\title{
Ultrastructural identification of telocytes in the muscularis of chicken ileum
}

\author{
PING YANG, YA'AN LIU, NISAR AHMED, SHAKEEB ULLAH, YI LIU and QIUSHENG CHEN \\ Key Laboratory of Animal Physiology and Biochemistry, Ministry of Agriculture, \\ College of Veterinary Medicine, Nanjing Agricultural University, Nanjing, Jiangsu 210095, P.R. China
}

Received November 13, 2014; Accepted September 24, 2015

DOI: 10.3892/etm.2015.2841

\begin{abstract}
Telocytes (TCs) are a specialized type of interstitial cells, characterized by a small cell body and long, thin processes, that have recently been identified in various cavitary and non-cavitary organs of humans and laboratory mammals. Chickens present significant economical and scientific notability; however, ultrastructural identification of TCs remains unclear in birds. The aim of the present study was to describe electron microscopic evidence for the presence of TCs in the chicken gut. The ileum of healthy adult broiler chickens $(n=10)$ was studied by transmission electron microscopy. TCs are characterized by several, long (tens to hundreds of $\mu \mathrm{m}$ ) prolongations called telopodes (Tps). Tps, which are below the resolving power of light microscopy, display podomeres (thin segments of $\leq 0.2 \mu \mathrm{m}$ ) and podoms (dilations accommodating caveolae, mitochondria and endoplasmic reticulum). TCs were observed in every field, but were predominantly located in the myenteric plexus and the lamina propria. Tps frequently establish close spatial relationships with immune cells, blood vessels and nerve endings. On the basis of their distribution and morphology, it was hypothesized that the different locations of TCs may be associated with different roles.
\end{abstract}

\section{Introduction}

The intestinal walls contain heterogeneous populations of stromal cells, including one of the most important stromal cells, the interstitial cells of Cajal (ICCs) (1). ICCs are a class of cells located between the autonomic nerve endings and smooth muscle cells in the gastrointestinal tract (GIT). ICCs produce a direct impact on the contraction of smooth muscle, such as peristalsis and segmentation in the GIT. The pacemaker activity of ICC cells may initiate myoelectric slow

Correspondence to: Professor Qiusheng Chen, Key Laboratory of Animal Physiology and Biochemistry, Ministry of Agriculture, College of Veterinary Medicine, Nanjing Agricultural University, Yifu building, 1 Tongwei road, Nanjing, Jiangsu 210095, P.R. China E-mail: chenqsh305@njau.edu.cn

Key words: telocytes, ultrastructure, ileum, chicken waves, a basal electrical rhythm leading to contraction of the smooth muscle (1-3). In the last decade, ICCs were investigated from a number of aspects, including ultrastructure, immunoreactivity, electrophysiology and pathology (4-6). In a 2008 study (7), the intestinal wall cell population was re-examined; consequently, a novel cell type was observed by transmission electron microscopy (TEM) and immunohistochemistry. This novel type of interstitial cell, previously known as interstitial Cajal-like cells (ICLCs), are now established to be telocytes (TCs) (8). Characteristics of TCs include long, projected prolongations termed telopodes (Tps) measuring tens to hundreds of $\mu \mathrm{m}$ long, as determined from TEM images (free access data are available at www.telocytes.com). Tps are podomeres with dilated portions termed podoms. TCs present no similarity to ICCs on the basis of structural differences.

TCs have been described in the interstitium of certain parenchimatous organs in mammals (9-22) and, although ICCs have been identified in the digestive tract of turkeys (23) and in the intestine of chickens (24), the presence of ICLCs or TCs in the digestive system of the chicken remains unclear. In addition, Cantarero Carmona et al (25) described TCs at the intestinal level, in the lamina propria of rat duodenum; however, to the best of our knowledge, studies on TCs in the intestinal muscularis have not previously been conducted.

Poultry has great economical importance as it is a major source of meat to fulfill the human requirement. The present study aimed to provide further details on the identification and features of TCs in the muscularis of chicken ileum. Their relationships with neighboring cells, including nerve cells and smooth muscle cells (SMC) in the muscularis of adult chicken ileum, were investigated.

\section{Materials and methods}

A total of 10 healthy adult Chinese Three Yellow broilers (English translation of the local Chinese chicken breed), between 7 and 9 weeks of age and weighing 1.8-2.0 kg/chicken, were used in the current study. The feeding was conducted with commercially available feeds (New Hope Group, Chengdu, China) for adult broiler chickens. They were housed in temperature-controlled rooms $\left(20 \pm 1^{\circ} \mathrm{C}\right)$ with natural light (light/dark cycle, 12/12 h), and had free access to food and tap water. The chickens were sacrificed by cervical dislocation, under $3 \%$ ether anesthesia through inhalation. The experiment was approved 


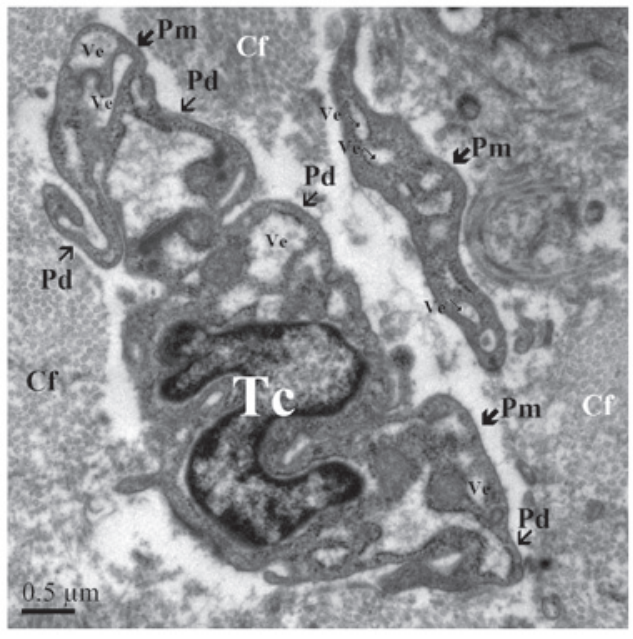

Figure 1. Transmission electron microscopy image of a clear Tc in the muscularis of chicken ileum. Tc cells are present with a small body and a variable number of telopodes, containing a nucleus, surrounded by a small volume of cytoplasm. Bars, $0.5 \mu \mathrm{m}$. Cf, collagen fiber; Pd, podomer; Pm, podom; $\mathrm{Tc}$, telocyte; Ve, vesicle.

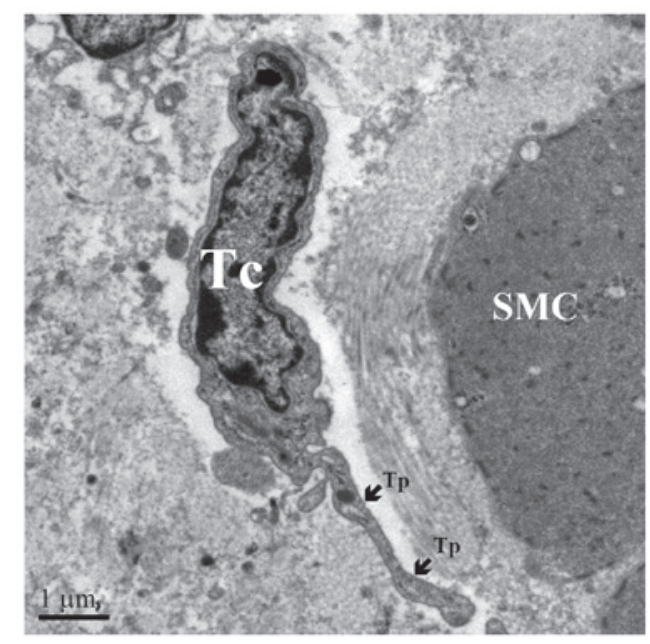

Figure 2. Transmission electron microscopy image of a Tc in the muscularis of chicken ileum. Tcs exhibit a piriform shape with 1 long prolongation, which is a Tp. Bars, $1 \mu \mathrm{m}$. SMC, smooth muscle cell; Tc, telocyte; Tp, telopode.

by the Science and Technology Agency of Jiangsu Province [license number, SYXK (SU) 2011-0036]. Tissue samples were collected from the ileum and immersed in $2.5 \%$ glutaraldehyde fixative (Sigma-Aldrich, Saint Louis, USA) in $0.1 \mathrm{M}$ phosphate-buffered saline $\left(4^{\circ} \mathrm{C}, \mathrm{pH}\right.$ 7.4) for $1 \mathrm{~h}$, then washed in the same buffer overnight. The tissues were postfixed with a mixture of $1 \%$ osmiumtetroxide (Sigma-Aldrich) and $1.25 \%$ potassium ferrocyanide (Sigma-Aldrich) for 1.5-2.0 h, washed in the buffer, dehydrated in increasing concentrations of ethylalcohol (75 and 85\%, for $1 \mathrm{~h}$ each; 95 and 100\%, two cycles for $1 \mathrm{~h}$ each), infiltrated with a propylene oxide/Araldite mixture (Boster, Wuhan, China), and embedded in Araldite (Boster). Semithin sections $(1 \mu \mathrm{m})$ of the intestine were labeled with $1 \%$ toluidine blue (Sigma-Aldrich) for light microscope examination, and specific areas were selected for ultrathin sectioning. Sections were cut using a Reichert Jung Ultracut E ultramicrotome (Reichert, Vienna, Austria). Ultrathin sections (50 nm)

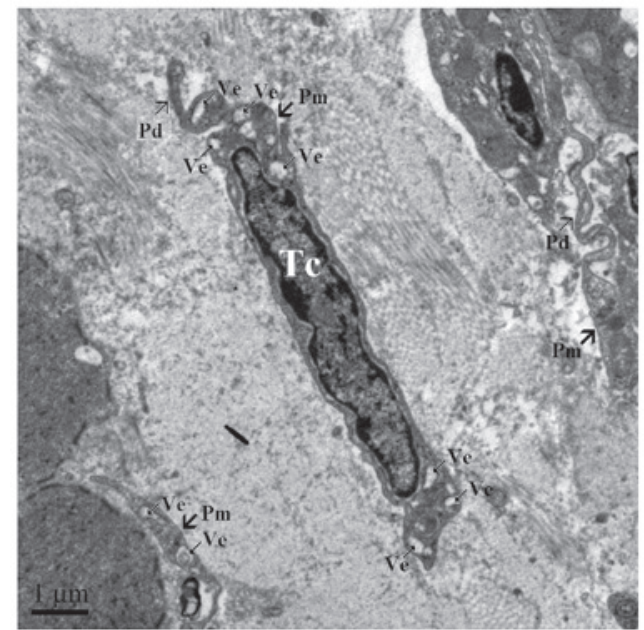

Figure 3. Transmission electron microscopy image of a Tc in the muscularis of chicken ileum. Tcs are prominent with a long spindle shape and with 2 telopodes. Ve are also clearly visible in the telopodes of Tcs. Bars, $1 \mu \mathrm{m}$. Pd, podomer; Pm, podom; Tc, telocyte; Ve, vesicle.

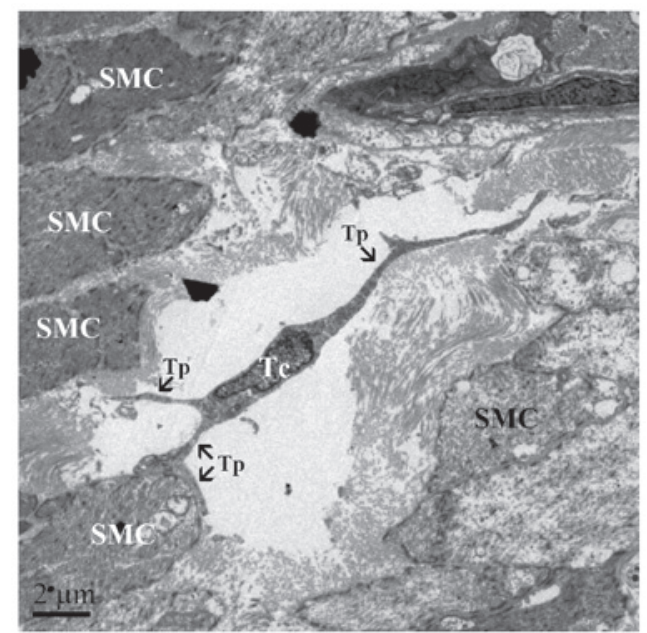

Figure 4. Transmission electron microscopy image of a Tc within the longitudinal and circular muscle layers of chicken ileum. Tc demonstrates a triangular shape with $3 \mathrm{Tp}$ attached from surroundings. Tcs are closely attached to smooth muscle cells through their Tps. Bars, $2 \mu \mathrm{m}$. SMC, smooth muscle cell; Tc, telocyte; Tp, telopode.

were collected on copper grids, counterstained for $10 \mathrm{~min}$ with $1 \%$ uranyl acetate (Sigma-Aldrich) and Reynold's lead citrate (Sigma-Aldrich), then observed with a JEM-1200EX transmission electron microscope (Jeol, Tokyo, Japan).

\section{Results}

TEM examination is fundamental for identifying TCs. TCs are cells with a small body and a variable number of Tps, containing a nucleus, and are surrounded by a small volume of cytoplasm (Fig. 1). Tps have a particular ultrastructural signature, consisting of alternating thin segments (podomers) and dilations (podoms). Each TC has 1-5 Tps, and the shape of the TC is determined by the number of their Tps: Piriform for 1 prolongation (Fig. 2), spindle for 2 Tps (Fig. 3), triangular for 3 and stellate for $\geq 4$ Tps (Fig. 4). The thin rim of cytoplasm 


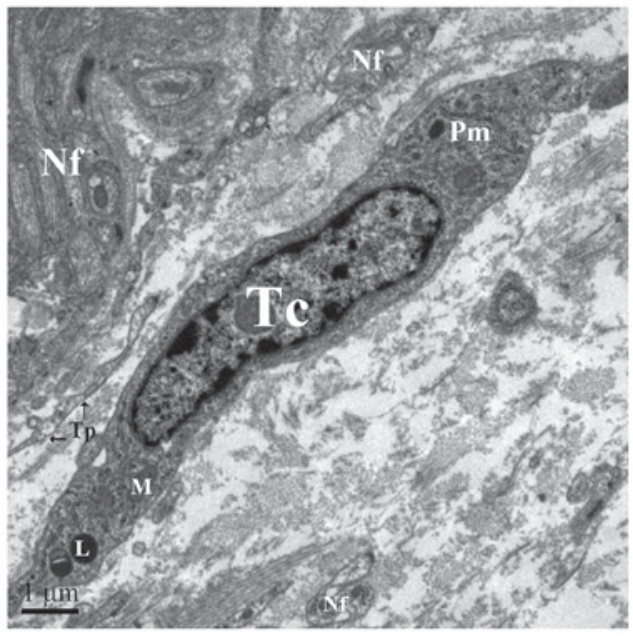

Figure 5. Transmission electron microscopy image of a Tc in the muscularis of chicken ileum. Tc shows the thin rim of cytoplasm surrounding the nucleus, contains a small Golgi apparatus, some mitochondria and few cisternae of rough endoplasmic reticulum and of smooth endoplasmic reticulum. Bars, $1 \mu \mathrm{m}$. L, lysosome; M, mitochondrion; Nf, nerve fiber; Pm, podomer; Tc, telocyte; Tp, telopode.

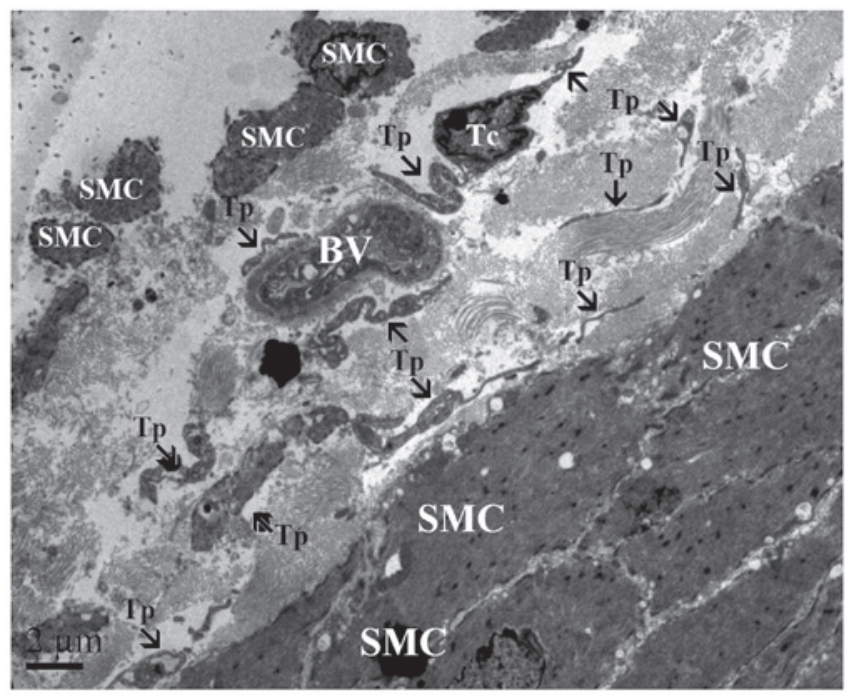

Figure 6. Transmission electron microscopy image of Tcs in the myenteric plexus of chicken ileum. The Tcs are in close contact with surrounding smooth muscle cells. Tps also clearly surround blood vessels. Bars, $1 \mu \mathrm{m}$. BV, blood vessel; SMC, smooth muscle cell; Tc, telocyte; Tp, telopode.

surrounding the nucleus contains a small Golgi apparatus, a number of mitochondria, and few cisternae of rough and smooth endoplasmic reticulum (Fig. 5).

In the myenteric plexus, TCs were identified according to a particular ultrastructural signature. They often surround blood vessels (Fig. 6) and certain TCs are connected to each other (Fig. 7) and neighboring SMCs (Fig. 6) via gap junctions. Bundles of enteric nerve fibers lay close to TCs (Fig. 8), although intimate relationships were not observed. In the current study, TCs were predominantly spindle-shaped, with 2 Tps.

Other TCs were located within the longitudinal and circular muscle layers, containing numerous mitochondria, caveolae and rough endoplasmic reticulum. These TCs displayed similar

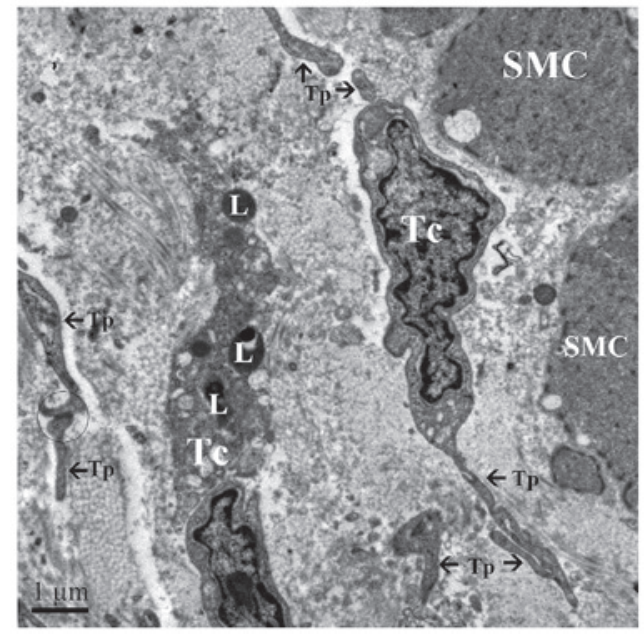

Figure 7. Transmission electron microscopy image of Tcs in the myenteric plexus of chicken ileum. Tcs shows clear contact with smooth muscle cells and blood vessels, aided by telopod. Some Tps gap junctions with each other (arrowheads). Bars, $2 \mu \mathrm{m}$. L, lysosome; SMC, smooth muscle cell; Tc, Telocyte; Tp, telopode.

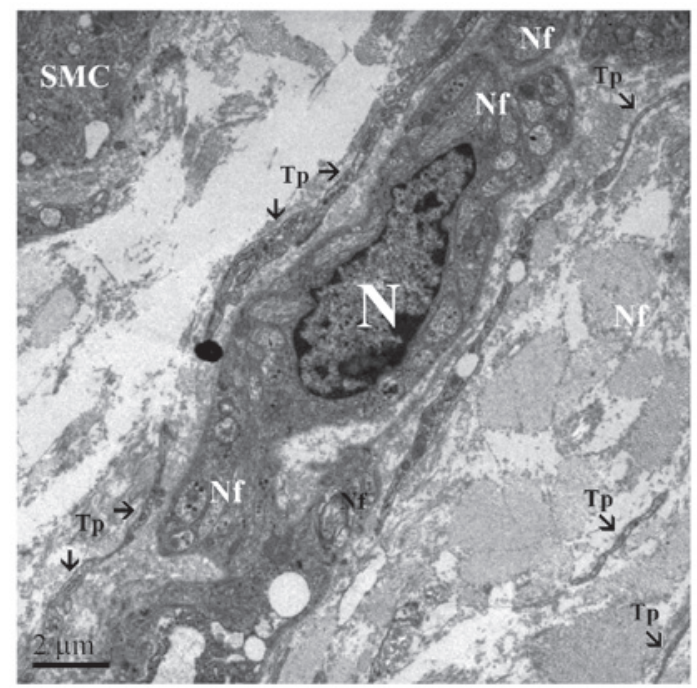

Figure 8. Transmission electron microscopy image of Tcs in the myenteric plexus of chicken ileum. The bundles of enteric nerve fibers are clearly visible around the Tcs. Bars, $2 \mu \mathrm{m}$. Nf, nerve fiber; SMC, smooth muscle cell; Tc, telocyte; Tp, telopode.

ultrastructural features, and exhibited a predominantly stellate shape with 2 long processes and multiple short processes (Fig. 4). The ends of the processes often formed large caveolae (Fig. 4). The TCs of the longitudinal layer formed distinct close contacts, not gap junctions, with adjacent SMCs (Fig. 4).

Numerous TCs were observed along the submucosal surface of the circular muscle layer. These TCs were arranged around the ganglia of the submucosal plexus (Fig. 9) and occasionally surrounded neighboring SMC (Fig. 10).

TCs were located in the lamina propria of the ileum. These cells presented a stellate morphology with multiple branches. The branches made frequent close contacts with each other, forming a network and close contacts with neighboring cells (Fig. 11). In addition, these TCs displayed longer Tps than the TCs in muscularis. 


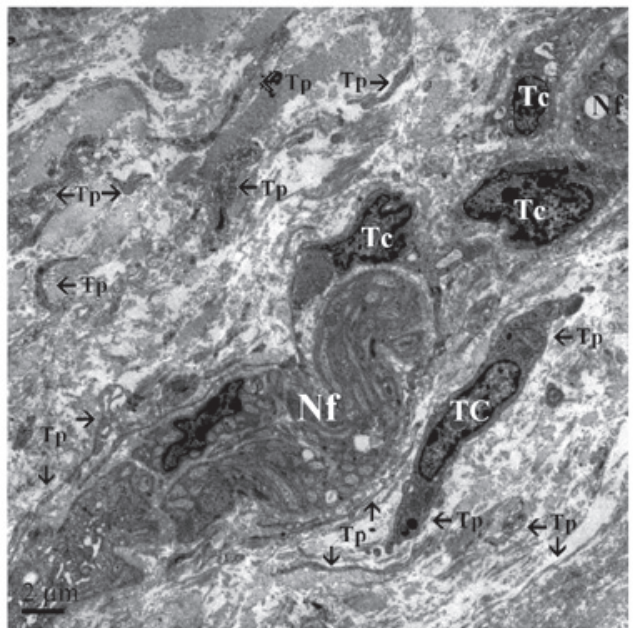

Figure 9. Transmission electron microscopy image of Tcs along the submucosal surface of the circular muscle layer in the chicken ileum. Tcs were arranged around the ganglia of the submucosal plexus. Bars, $2 \mu \mathrm{m}$. Nf, nerve fiber; Tc, telocyte; Tp, telopode.

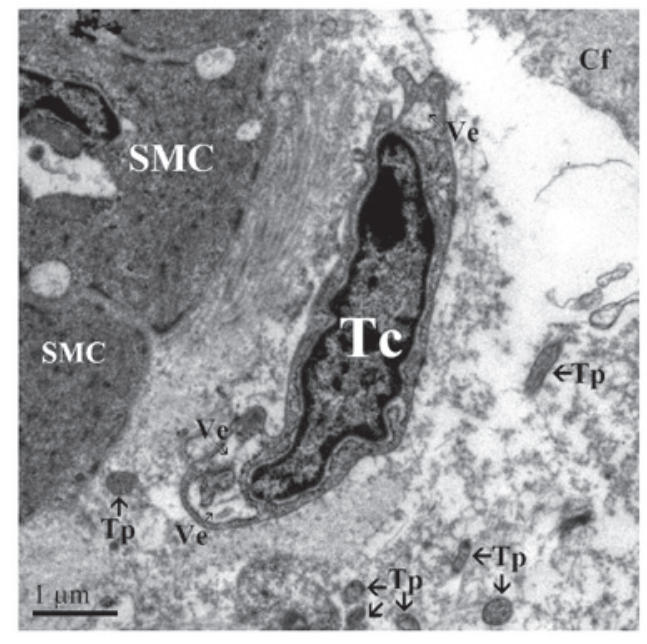

Figure 10. Transmission electron microscopy image of Tcs surrounding neighboring SMCs in the submucosal plexus of the chicken ileum. Bars, $2 \mu \mathrm{m}$. Nf, nerve fiber; SMC, smooth muscle cell; Tc, telocyte; Tp, telopode; Ve, vesicle.
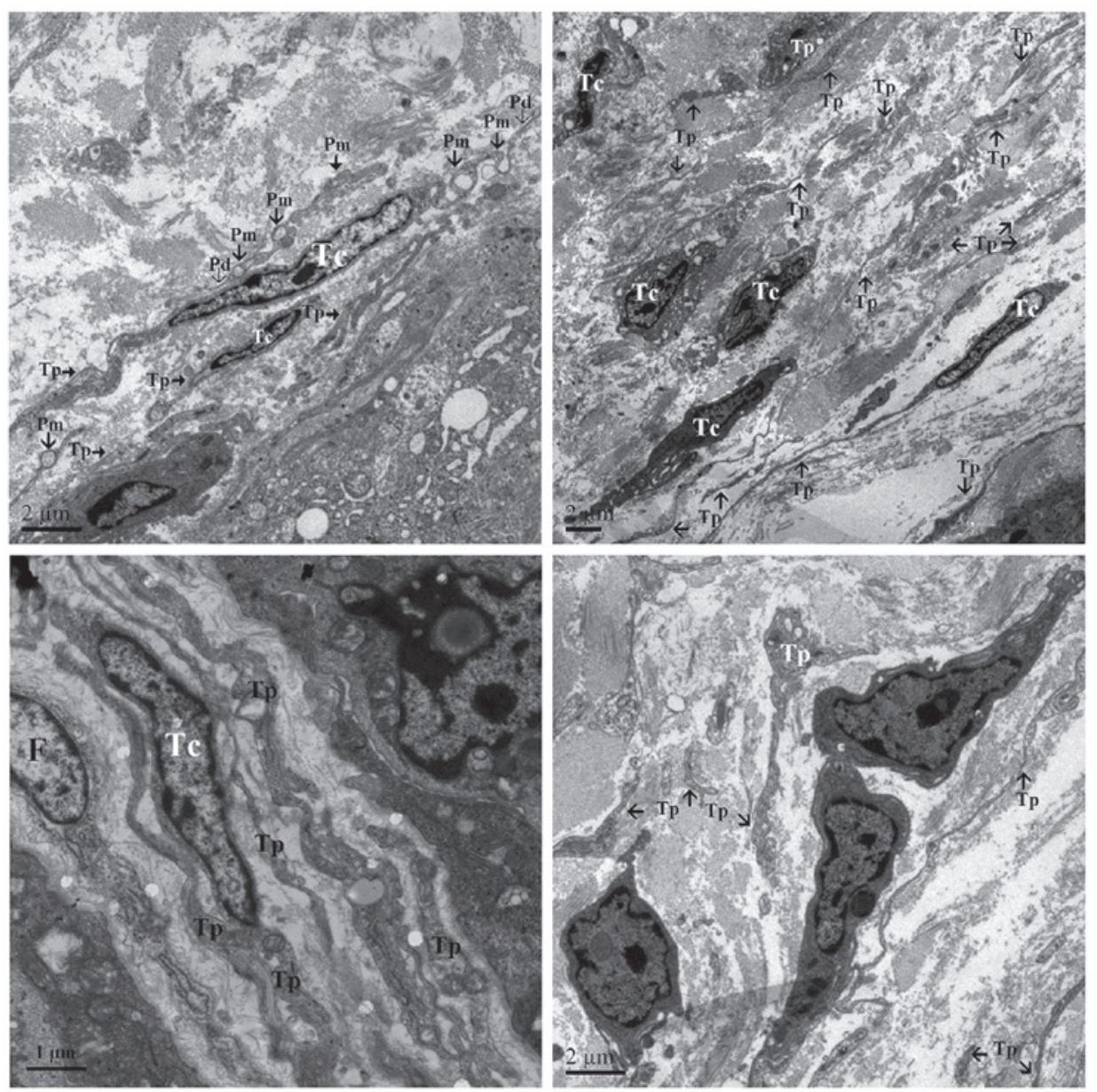

Figure 11. Transmission electron microscopy image of Tcs in the lamina propria of the chicken ileum. These cells had a stellate morphology with multiple branches. The branches made frequent close contacts with each other, forming a network and close contact with neighboring cells. F, fibroblast; N, nucleus; Nf, nerve fiber; Pd, podomer; Pm, podom; SMC, smooth muscle cell; Tc, telocyte; Tp, telopode.

\section{Discussion}

The current study demonstrated the morphological and ultrastructural characteristics of TCs in the in the muscularis and the lamina propria of the chicken ileum, revealing characteristic ultrastructural features according to the TEM diagnostic criteria for TCs (8). Although the term 'telocyte' is relatively new, a review of the literature indicated various studies 
reporting the presence of TCs in other organs, including the epicardium (20), myocardium (21), endocardium (26), placenta (27) and skin (28). The role of TCs is unclear; however, based on the available data, numerous potential and relevant roles have been proposed. Taking into account the 3D network of Tps, and their strategic position between target cells, nerve endings and blood capillaries, Popescu and Faussone-Pellegrini (8) suggested that TCs may be involved in intercellular signaling. They considered at least two mechanisms that may be involved: i) Aparacrine and/or juxtacrine secretion of small signal molecules; and ii) shedding microvesicles, which serve unique roles in the horizontal transfer of important macromolecules among neighboring cells (e.g. proteins or RNAs, such as microRNA). The result of the present study also demonstrates a close connection of TCs with blood capillaries and nerve endings. It is suggested that TCs may have the following functions in the myocardium: i) Involvement in intercellular signaling at a distance, as they are situated close to blood capillaries and nerve endings; ii) mechanoreceptors/transducers, due to their extremely long Tps and their ability to form attachment plaques connecting them to the extracellular matrix; and iii) cardiac renewal and cardiac repair (29).

Cretoiu and Popescu described the close contacts that establish TCs with various types of immunoreactive cells, including lymphocytes, plasma cells, eosinophils, basophils, macrophages and mast cells in human mammary gland and myometrium, rat stomach, gut, bladder, and uterus (30). The current study demonstrated these contacts with immune cells in the lamina propria of chicken ileum; on the basis of contact distribution and morphology, these cells may be involved in the immune response.

The present study indicated that TCs form contacts with stem cells (SCs) in discrete sites that appear to be SC niches in the myenteric plexus. The tandem TC-SC has previously been observed in SC niches in a number of organs, including the epicardium, lungs, skeletal muscle, choroid plexus and skin (31). SC niches are highly organized interactive structural units that commonly occur at tissue intersections or transition zones, and coordinate tissue repair and renewal $(32,33)$. The functionality of a SC niche relies on the physical contact and signaling interactions of SCs with neighboring nurse cells, in addition to the paracrine and endocrine signals from local or distant sources, neural input and metabolic products of tissue (33). TCs have a strategic position in the ileum between blood capillaries and SC, and are in close contact with nerve endings. TCs may be nurse cells, integrating local, short-distance signals (direct contacts, exosomes and shedding vesicles) and long-distance signals through the long TPs, due to their 3D network. Recent studies have demonstrated a particular immunophenotype $(34,35)$, distinct microRNA expression (36), specific gene-expression profiles (37) and peculiar electrophysiological properties of TCs in various organs (38). Close contact between TCs and fibroblasts was indicated in the present study. Fibroblasts can be differentiated from TCs on the basis of their short cell processes with thick protrusions from the cell body. Furthermore, they exhibit a different phenotype from Tps, which are long, moniliform and convoluted (34). A comparative proteomic analysis of human lung TCs versus fibroblasts showed that TCs are different from fibroblasts (39). The results described here are in agreement with these findings as there are clear long, moniliform and convoluted Tps.

The function of TCs in chicken ileum may be associated with the reparation of injured tissues during disease, as in skeletal muscle (18). This hypothesis is supported by the present observation that TCs and Tps were located in close vicinity to blood capillaries, nerve endings and smooth muscle cells. However, there is a requirement for the investigation of TC-specific biomarkers in order to clarify TC cell functionality and network biomarkers, to improve understanding of the interaction between proteins, genes, signaling pathways and dynamic networks, and to define and predict time-dependent TC function and morphological features $(40,41)$. TCs also function in the vascular system, nervous system, immune system, interstitium and $\mathrm{SC}$ /progenitors (42). Manole et al (36) demonstrated that TCs are involved in neo-angiogenesis in experimental acute myocardial infarction. TCs may further be involved in tissue regeneration and reparation $(28,18)$; it is suggested that TCs are involved in angiogenesis, as it has been demonstrated that TCs express CD34 and VEGF (27).

The present study provided ultrastructural evidence for the existence of TCs in the muscularis and the lamina propria of the chicken ileum. Tps connect with immune cells, smooth muscle cells, nerve fibers and blood vessels. These data demonstrate the existence of TCs in birds. Future studies should explore the potential biological functions of TCs in certain pathological conditions of the intestine, and investigate the mechanisms of interaction between TCs and other cells.

\section{Acknowledgements}

The present study was supported by grants from the National Natural Science Foundation of China (Youth Project, no. 31402155 and no. 31172282), the Natural Science Foundation of Jiangsu Province of China (youth project, no. BK20130681), the Fundamental Research Funds for the Central Universities of China (no. KJ201302), and the Priority Academic Program Development of Jiangsu Higher Education Institutions of China.

\section{References}

1. Faussone-Pellegrini MS and Thuneberg L: Guide to the identification of interstitial cells of Cajal. Microsc Res Tech 47: 248-266, 1999.

2. Daniel EE: Physiology and pathophysiology of the interstitial cell of Cajal: From bench to bedside. III. Interaction of interstitial cells of Cajal with neuromediators: an interim assessment. Am J Physiol Gastrointest Liver Physiol 281: G1329-G1332, 2001.

3. Ward SM and Sanders KM: Interstitial cells of Cajal: Primary targets of enteric motor innervation. Anat Rec 262: 125-135, 2001.

4. Vanderwinden JM, Rumessen JJ, Bernex F, Schiffmann SN and Panthier JJ: Distribution and ultrastructure of interstitial cells of Cajal in the mouse colon, using antibodies to Kit and Kit(W-lacZ) mice. Cell Tissue Res 302: 155-170, 2000.

5. Farrugia G: Interstitial cells of Cajal in health and disease. Neurogastroenterol Motil 20 (Suppl 1): 54-63, 2008.

6. Mikkelsen HB: Interstitial cells of Cajal, macrophages and mast cells in the gut musculature: Morphology, distribution, spatial and possible functional interactions. J Cell Mol Med 14: 818-832, 2010 . 
7. Pieri L, Vannucchi MG and Faussone-Pellegrini MS Histochemical and ultrastructural characteristics of an interstitial cell type different from ICC and resident in the muscle coat of human gut. J Cell Mol Med 12: 1944-1955, 2008.

8. Popescu LM and Faussone-Pellegrini MS: Telocytes - a case of serendipity: The winding way from interstitial cells of Cajal (ICC), via interstitial Cajal-like cells (ICLC) to telocytes. J Cell Mol Med 14: 729-740, 2010.

9. Nicolescu MI and Popescu LM: Telocytes in the interstitium of human exocrine pancreas: Ultrastructural evidence. Pancreas 41 : 949-956, 2012.

10. Díaz-Flores L, Gutiérrez R, Sáez FJ, Díaz-Flores L Jr and Madrid JF: Telocytes in neuromuscular spindles. J Cell Mol Med 17: 457-465, 2013

11. Milia AF, Ruffo M, Manetti M, Rosa I, Conte D, Fazi M, Messerini L, Ibba-Manneschi L: Telocytes in Crohn's disease. J Cell Mol Med 17: 1525-1536, 2013.

12. Manetti M, Rosa I, Messerini L, Guiducci S, Matucci-Cerinic M and Ibba-Manneschi L: A loss of telocytes accompanies fibrosis of multiple organs in systemic sclerosis. J Cell Mol Med 18 253-262, 2014.

13. Li L, Lin M, Li L, Wang R, Zhang C, Qi G, Xu M, Rong R and Zhu T: Renal telocytes contribute to the repair of ischemically injured renal tubules. J Cell Mol Med 18: 1144-1156, 2014.

14. Li H, Zhang H, Yang L, Lu S and Ge J: Telocytes in mice bone marrow: Electron microscope evidence. J Cell Mol Med 18 975-978, 2014

15. Nicolescu MI and Manole CG: Telocytes and stem cells in regenerative medicine. FASEB J 27: 752-754, 2013.

16. Chen X, Zheng Y, Manole CG, Wang X and Wang Q: Telocytes in human oesophagus. J Cell Mol Med 17: 1506-1512, 2013.

17. Popescu BO, Gherghiceanu M, Kostin S, Ceafalan L and Popescu LM: Telocytes in meninges and choroid plexus. Neurosci Lett 516: 265-269, 2012

18. Popescu LM, Manole E, Serboiu CS, Manole CG, Suciu LC, Gherghiceanu M and Popescu BO: Identification of telocytes in skeletal muscle interstitium: implication for muscle regeneration. J Cell Mol Med 15: 1379-1392, 2011.

19. Popescu LM, Gherghiceanu M, Suciu LC, Manole CG and Hinescu ME: Telocytes and putative stem cells in the lungs: Electron microscopy, electron tomography and laser scanning microscopy. Cell Tissue Res 345: 391-403, 2011.

20. Popescu LM, Manole CG, Gherghiceanu M, Ardelean A, Nicolescu MI, Hinescu ME and Kostin S: Telocytes in human epicardium. J Cell Mol Med 14: 2085-2093, 2010.

21. Kostin S: Myocardial telocytes: A specific new cellular entity. J Cell Mol Med 14: 1917-1921, 2010.

22. Bani D, Formigli L, Gherghiceanu M and Faussone-Pellegrini MS: Telocytes as supporting cells for myocardial tissue organization in developing and adult heart. J Cell Mol Med 14: 2531-2538, 2010.

23. Reynhout JK and Duke GE: Identification of interstitial cells of Cajal in the digestive tract of turkeys (Meleagris gallopavo). J Exp Zool 283: 426-440, 1999.

24. Yang P, Wang S, Gandahi J, Bian X, Wu L, Liu Y, Zhang L, Zhang Q and Chen Q: Ultrastructural identification of different subtypes of interstitial cells of Cajal in the chicken ileum. Poult Sci 91: 1936-1940, 2012.
25. Cantarero Carmona I, Luesma Bartolomé MJ and Junquera Escribano C: Identification of telocytes in the lamina propria of rat duodenum: transmission electron microscopy. J Cell Mol Med 15: 26-30, 2011.

26. Gherghiceanu M, Manole C and Popescu L: Telocytes in endocardium: Electron microscope evidence. J Cell Mol Med 14: 2330-2334, 2010.

27. Suciu L, Popescu LM, Gherghiceanu M, Regalia T, Nicolescu MI, Hinescu ME and Faussone-Pellegrini MS: Telocytes in human term placenta: Morphology and phenotype. Cells Tissues Organs 192: 325-339, 2010.

28. Ceafalan LM, Gherghiceanu M, Popescu L and Simionescu O: Telocytes in human skin-are they involved in skin regeneration? J Cell Mol Med 16: 1405-1420, 2012.

29. Kostin S and Popescu L: A distinct type of cell in myocardium: Interstitial Cajal-like cells (ICLCs). J Cell Mol Med 13: 295-308, 2009.

30. Cretoiu SM and Popescu LM: Telocytes revisited. Biomol concepts 5: 353-369, 2014

31. Popescu LM and Nicolescu MI: Telocytes and stem cells. In Resident Stem Cells and Regenerative Therapy. Goldenberg and Campos de Carvalho (eds). Elsevier, Amsterdam, pp205-231, 2013.

32. Ordonez P and Di Girolamo N: Limbal epithelial stem cells: Role of the niche microenvironment. Stem Cells 30: 100-107, 2012.

33. Scadden DT: The stem-cell niche as an entity of action. Nature 441: 1075-1079, 2006.

34. Faussone-Pellegrini MS and Popescu LM: Telocytes. Biomol Concepts 2: 481-489, 2011.

35. Koh BH, Roy R, Hollywood MA, Thornbury KD, McHale NG, Sergeant GP, Hatton WJ, Ward SM, Sanders KM and Koh SD: Platelet-derived growth factor receptor- $\alpha$ cells in mouse urinary bladder: a new class of interstitial cells. J Cell Mol Med 16: 691-700, 2012.

36. Manole CG, Cismaşiu V, Gherghiceanu M and Popescu LM: Experimental acute myocardial infarction: Telocytes involvement in neo-angiogenesis. J Cell Mol Med 15: 2284-2296, 2011.

37. Zheng Y, Zhang M, Qian M, Wang L, Cismasiu VB, Bai C, Popescu LM and Wang X: Genetic comparison of mouse lung telocytes with mesenchymal stem cells and fibroblasts. J Cell Mol Med 17: 567-577, 2013.

38. Cretoiu SM, Cretoiu D, Marin A, Radu BM and Popescu LM: Telocytes: Ultrastructural, immunohistochemical and electrophysiological characteristics in human myometrium. Reproduction 145: 357-370, 2013

39. Zheng Y, Cretoiu D, Yan G, Cretoiu SM, Popescu LM and Wang X: Comparative proteomic analysis of human lung telocytes with fibroblasts. J Cell Mol Med 18: 568-589, 2014

40. Chen H and Wang X: Significance of bioinformatics in research of chronic obstructive pulmonary disease. J Clin Bioinforma 1: 35, 2011.

41. Wu D, Rice CM and Wang X: Cancer bioinformatics: A new approach to systems clinical medicine. BMC Bioinformatics 13: 71, 2012.

42. Gherghiceanu M and Popescu LM: Cardiac telocytes - their junctions and functional implications. Cell Tissue Res 348: 265-279, 2012 